\title{
Assistência Odontológica Pública e Suplementar no Município de São Paulo na Primeira Década do Século XXI
}

\section{Private and Public Dental Care in the City of São Paulo in the}

\section{First Decade of the XXI Century}

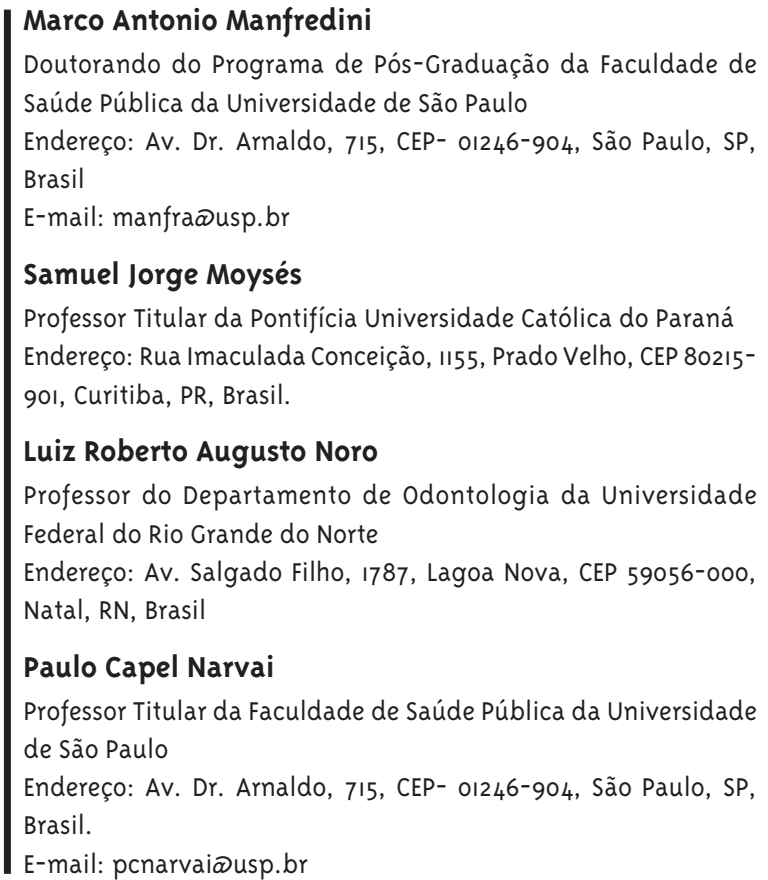

\section{Luiz Roberto Augusto Noro}

Professor do Departamento de Odontologia da Universidade Federal do Rio Grande do Norte

Endereço: Av. Salgado Filho, 1787, Lagoa Nova, CEP 59056-000, Natal, RN, Brasil

\section{Paulo Capel Narvai}

Professor Titular da Faculdade de Saúde Pública da Universidade de São Paulo

Endereço: Av. Dr. Arnaldo, 715, CEP- 01246-904, São Paulo, SP, Brasil.

E-mail: pcnarvaiळusp.br

\section{Resumo}

Na primeira década do século XXI registra-se a ocorrência de dois movimentos importantes no âmbito da assistência odontológica pública e privada no Brasil: a entrada da saúde bucal na agenda de prioridades políticas do governo federal e o vigoroso crescimento na oferta de serviços odontológicos suplementares. Analisou-se a ocorrência desses fenômenos no município de São Paulo, mediante a busca de dados nos documentos oficiais e nas bases eletrônicas da Prefeitura Municipal de São Paulo, do Ministério da Saúde e da Agência Nacional de Saúde Suplementar (ANS), além de consulta à literatura científica. No período estudado, de janeiro de 2000 a dezembro de 2009, com base em indicadores como a Cobertura de Primeira Consulta Odontológica Programática e a Cobertura Populacional Potencial, verificaram-se percentuais que caracterizam baixa assistência pública e uma situação de grande distanciamento do princípio constitucional do acesso universal aos cuidados odontológicos. 0 crescimento do número de beneficiários de serviços suplementares, por meio de planos exclusivamente odontológicos e de outros planos foi expressivo em igual período, correspondendo a uma importante ampliação da cobertura populacional nesta modalidade assistencial. Constata-se que, comparativamente ao quadro geral nacional, a situação do município de São Paulo revela precariedade no acesso à assistência odontológica pública, com reduzida oferta de serviços a adultos e idosos. Considerando, ainda, as limitações do mercado de serviços suplementares para prover assistência odontológica para todos os brasileiros, reforça-se a necessidade de continuidade e expansão 
do Brasil Sorridente, que é a expressão programática da Política Nacional de Saúde Bucal.

Palavras-chave: Políticas Públicas de Saúde; Acesso aos Serviços de Saúde; Serviços de Saúde Bucal.
Abstract

Two concomitant movements occur in the first decade of the XXI century within the private and public dental services in Brazil: the entrance of oral health on the agenda of political priorities of the federal government and the vigorous growth of additional dental care. We analyzed the occurrence of these phenomena in the city of São Paulo, by seeking information in official documents and electronic databases in the Municipality of São Paulo, the Ministry of Health and National Health Agency (ANS), and also in scientific literature. During the studied period - January 2000 to December 2009 - and with basis on indicators such as coverage of First Consultation Program and Dental coverage Population Potential, percentages were found that characterize low public assistance and a situation far short of the constitutional principle of universal access to dental care. The growing number of beneficiaries of additional services through exclusively dental coverage insurance plans and other types of private insurance plans in the same period was significant, accounting for a major expansion of population coverage in this mode of care. It was found that, compared to the overall national framework, the city of São Paulo offers poor access to public dental care, with reduced supply of services to adults and aged people. Furthermore, considering the limitations of market additional services to provide dental care to all Brazilians, it reinforces the need for continuity and expansion of Brasil Sorridente, which is the programmatic expression of the National Oral Health Politics.

Keywords: Public Health Policies; Health Services Accessibility; Dental Health Services. 


\section{Introdução}

Ao término da primeira década do século XXI, a odontologia brasileira consolida quantitativamente sua posição como uma das mais importantes no cenário mundial. 0 país dispunha de 193 faculdades de odontologia e cerca de 229 mil cirurgiões-dentistas estavam inscritos em 2010 nos conselhos que fiscalizam o exercício profissional (CFO, s.d.).

O complexo odontológico-industrial é pujante, com predomínio de empresas com maior aporte de capital nacional no setor, que respondem pela produção de equipamentos de ponta, com razoável grau de acúmulo tecnológico e posição de destaque no mercado global. o país é o segundo maior mercado de produtos de higiene bucal no mundo, constituindo-se em uma grande plataforma produtora e exportadora desses insumos (Manfredini, 2006; 2009b).

O quadro epidemiológico indica uma transição positiva, com redução dos níveis de cárie em crianças. Contudo, persistem iniquidades regionais e uma importante carga de doenças bucais em adultos e idosos, observando-se melhores condições nas áreas urbanas de capitais do Sul e Sudeste. Em decorrência da denominada transição demográfica, doenças que acometem mais os idosos, como o câncer de boca, ganham importância crescente, embora não haja evidência de aumento significativo nos coeficientes de incidência.

Dois fenômenos concomitantes ocorrem nesta década, no âmbito dos serviços odontológicos públicos e privados brasileiros: a entrada da saúde bucal na agenda de prioridades políticas do governo federal e o vigoroso crescimento da assistência odontológica suplementar. Neste artigo, verifica-se como proposição geral a ocorrência e eventuais implicações desses fenômenos no âmbito do município de São Paulo, maior cidade do país, destacando-se o objetivo de alinhar suas práticas públicas odontológicas à Política Nacional de Saúde Bucal.

\section{Métodos}

Procedeu-se a uma busca de dados nos documentos oficiais e nas bases eletrônicas da Prefeitura Municipal de São Paulo, do Ministério da Saúde e da Agên- cia Nacional de Saúde Suplementar (ANS), além de consulta à literatura científica. O período de estudo analisado foi de janeiro de 2000 a dezembro de 2009. O estudo se valeu, portanto, de dados secundários, obtidos a partir da coleta e análise das informações obtidas nos documentos, em bases eletrônicas e junto a órgãos dessas instituições.

O município de São Paulo é o maior do país, em termos demográficos e econômicos. Concentra 5,7\% da população moradora no Brasil e $26,2 \%$ da residente no Estado de São Paulo. Responde por 12\% do Produto Interno Bruto (PIB) do país e por mais de 35\% daquele produzido no Estado, sendo superior ao de qualquer Estado brasileiro, com exceção do próprio Estado de São Paulo (Fundação Seade, 2010). A população do município cresce na atualidade a taxas de $0,59 \%$ ao ano, valor correspondente a apenas $10 \%$ do registrado na década de 1950 (5,58\%). Observa-se a redução de pessoas por domicílio, de 4,8 em 1950 para 3,1 em 2010. O padrão etário da população ficou mais maduro, com o dobro da população de idosos no total da população e diminuição de $5 \%$ nos jovens com menos de 15 anos de idade (Fundação Seade, 2010).

A cidade caracteriza-se pelos contrastes e pela heterogeneidade socioespacial. Com mais de 11 milhões de habitantes, é o município mais rico do país, mas tem aproximadamente 990 mil pessoas residindo em áreas de pobreza. As suas áreas centrais e seu entorno exibem indicadores socioeconômicos mais favoráveis que as áreas periféricas. São Paulo conta com 31 subprefeituras, sendo que as localizadas na periferia apresentam altas taxas de crescimento populacional, com maiores taxas de mortalidade infantil e menor longevidade que as áreas centrais e seus entornos (São Paulo, 2007b).

A cidade é o polo da Região Metropolitana de São Paulo (RMSP). Um em cada dez brasileiros vive na RMSP. A região oferta aproximadamente 5,5 milhões de empregos, que representam pouco mais de um sexto dos empregos nacionais. Com uma participação de $15,6 \%$ sobre o PIB nacional, a RMSP concentra pouco mais da metade do PIB do Estado, equivalendo ao produzido pelo Chile (Emplasa, 2007).

Rolnik (2004) destaca que, mesmo com a queda da participação relativa do município na produção industrial da RMSP e do estado, a cidade manteve- 
se como grande e dinâmico centro industrial. A indústria passou por um processo de reconversão, com a modernização tecnológica e gerencial das empresas mais capitalizadas, transnacionais e globalizadas, associada à precarização dos vínculos empregatícios e das condições de trabalho nas micro e pequenas unidades. A cidade sedia a maior parte da rede de gestão dos serviços financeiros e de prestação de serviços no país. Em 2003, a cidade concentrava $12,7 \%$ dos serviços de viagens e transportes; $12,5 \%$ da educação; $15,6 \%$ do consumo de serviços odontológicos; e 13,7\% de planos e seguros de saúde do país.

A partir da análise dos quatro indicadores de saúde bucal que compõem o Pacto de Atenção Básica de 2006, foi identificado o indicador "Cobertura de Primeira Consulta Odontológica Programática” para utilização neste estudo, para aferir o acesso a serviços odontológicos públicos. Este indicador aponta o percentual de pessoas que receberam uma primeira consulta odontológica programática, com a finalidade de diagnóstico e, necessariamente, a elaboração de um plano preventivo-terapêutico, para atender as necessidades detectadas e posterior realização do tratamento odontológico restaurador no âmbito da atenção básica, em determinado local e período. Neste indicador, não são referidos atendimentos eventuais como os de urgência/emergência que não tem seguimento previsto. Difere, portanto, do indicador "Cobertura de Primeira Consulta de Odontologia”, utilizado até 2005, que incluía os atendimentos eventuais de urgência.

Optou-se pela busca desta informação no Boletim CEInfo em Dados, da Coordenação de Epidemiologia e Informação da Secretaria Municipal de Saúde de São Paulo (São Paulo, 2009; Boletim..., 2008, 2010). Esta opção metodológica decorre do fato de que este instrumento é o utilizado para divulgação de informações de saúde e de apoio aos gestores do SUS pela referida Secretaria. Além disto, é disponibilizado em meio eletrônico e distribuído nas prestações de contas realizadas pelo Executivo municipal ao Conselho Municipal de Saúde e à Câmara Municipal. As informações disponibilizadas pela Coordenação Municipal de Saúde Bucal da Secretaria Municipal de Saúde e as acessadas pelo Sistema de Informações Ambulatoriais do SUS apresentaram diferenças numéricas de pequeno valor, em relação às disponibilizadas no Boletim CEInfo. Tal fato, entretanto, não interfere nesse estudo, pois a fonte de dados adotada pela Secretaria Municipal de Saúde em comunicações oficiais públicas é o Boletim CEInfo em Dados.

O Boletim CeInfo registra a primeira consulta odontológica programática a partir de 2006. Os registros de 2004 e 2005 se referem a primeiras consultas de odontologia, onde se incluíam os atendimentos de urgência realizados na rede pública municipal de saúde. Nos anos anteriores a 2003, não há registros das primeiras consultas de odontologia, no Boletim CEInfo.

Tendo em vista a inexistência de indicador específico sobre cobertura populacional produzida na esfera pública, criou-se um indicador que é apresentado neste estudo (Cobertura Populacional Potencial). Este indicador pôde ser construído, a partir de consulta ao sítio eletrônico da Coordenação Nacional de Saúde Bucal (CNSB) do Ministério da Saúde, onde há informações sobre o total de equipes de saúde bucal das modalidades I e II na Estratégia de Saúde da Família. A partir da soma das populações potencialmente cobertas por cada equipe, obtêm-se os valores da referida cobertura, que constam nos registros disponibilizados pela CNSB. Este indicador difere do cálculo de cobertura potencial, proposto por Chaves e Vieira-da-Silva (2007), que estabelece uma relação de um cirurgião-dentista para cada 3 mil habitantes com renda insuficiente, e dos indicadores "Cobertura Potencial de Recursos Humanos" e "Taxa de Cobertura Real de Procedimentos", propostos por Ribeiro-Sobrinho e colaboradores (2008). 0 indicador "Cobertura Populacional Potencial" não estabelece relação entre cirurgiões-dentistas e população e não analisa a capacidade de produção de procedimentos.

Na esfera da produção de serviços suplementares, considerou-se a Lei Federal $n^{0}$ 9.656/1998 e a Medida Provisória $n^{0}$ 2.177-44/2001 que definem "Operadora de Plano de Assistência à Saúde", como: "a pessoa jurídica constituída sob a modalidade de sociedade civil ou comercial, cooperativa, ou entidade de autogestão, que opere produto, serviço ou contrato de prestação continuada de serviços ou cobertura de custos assistenciais a preço pré 
ou pós estabelecido, por prazo indeterminado, com a finalidade de garantir, sem limite financeiro, a assistência à saúde, pela faculdade de acesso e atendimento por profissionais ou serviços de saúde, livremente escolhidos, integrantes ou não de rede credenciada, contratada ou referenciada, visando à assistência médica, hospitalar e odontológica, a ser paga integral ou parcialmente às expensas da operadora contratada, mediante reembolso ou pagamento direto ao prestador, por conta e ordem do consumidor" (Brasil, 1998, 2001).

A segmentação estabelecida pela legislação definiu como possibilidades de cobertura assistencial os planos: ambulatorial e odontológico; hospitalar com obstetrícia e odontológico; hospitalar sem obstetrícia e odontológico; hospitalar com obstetrícia, ambulatorial e odontológico e hospitalar sem obstetrícia, ambulatorial e odontológico. Os planos exclusivamente odontológicos são os que oferecem apenas assistência odontológica. As operadoras de planos privados de saúde exclusivamente odontológicas são agrupadas em cooperativa odontológica e odontologia de grupo. Segundo a Agência Nacional de Saúde Suplementar (ANS), o termo beneficiário se aplica a pessoa que possui vínculo a um determinado plano de saúde de uma operadora de plano privado de saúde.

Para este estudo, foram utilizadas as informações da ANS sobre o setor da saúde suplementar, por meio do Caderno de Informação da Saúde Suplementar e de acesso a informações disponibilizadas por órgãos da agência.

\section{Resultados}

A produção científica publicada em bases indexadas como Scielo, Pubmed e Lilacs, sobre acesso e utilização de serviços odontológicos, públicos e privados, no município de São Paulo é escassa, sendo mais encontráveis estudos sobre acesso e utilização geral de serviços de saúde na cidade (Louvison e col., 2008; Pessoto e col., 2007).

Documentos oficiais (São Paulo, 2010a) indicam que, em janeiro de 2010, a rede municipal de saúde possuía 434 Unidades Básicas de Saúde (UBS), sendo que 355 (81,7\%) dispunham de atendimento odontológico. Em 2004, eram 303. O município tinha 29 clínicas de especialidades odontológicas, diante de 17 em 2004. Destas clínicas, apenas três estavam cadastradas no Ministério da Saúde (MS) como Centros de Especialidades Odontológicas CEO (Brasil, 2010). O atendimento odontológico também era prestado em 14 serviços odontológicos localizados nos Serviços de Atendimento Especializado em DST/AIDS e 19 serviços em hospitais, pronto-atendimentos e prontos-socorros. O total de cirurgiões-dentistas na rede municipal era de 1.691, sendo que havia 841 auxiliares em saúde bucal e 157 técnicos em saúde bucal (São Paulo, 2010c).

O número de primeiras consultas de odontologia e primeiras consultas odontológicas programáticas, realizadas respectivamente em 2004 e 2005 e de 2006 a 2009, está indicado nas Tabelas 1 e 2. Ressalte-se que na Tabela 1, por se referirem às "primeiras consultas de odontologia”, estão incluídos atendimentos eventuais de urgência.

\section{Tabela I - Número de primeiras consultas de odontologia, população do município de São Paulo e porcentagem da cobertura, no período de 2004 e 2005}

\begin{tabular}{cccc} 
Ano & População do município & Primeiras consultas de odontologia & Cobertura de primeiras consultas de odontologia (\%) \\
\hline 2004 & 10.697 .738 & 202.160 & 1,9 \\
2005 & 10.766 .673 & 414.301 & 3,8 \\
\hline
\end{tabular}

Fonte: Secretaria Municipal de Saúde de São Paulo 
Tabela 2 - Número de primeiras consultas odontológicas programáticas, população do município de São Paulo e cobertura de primeira consulta odontológica programática, no período de 2006 a 2009

\begin{tabular}{cccc} 
Ano & População do município & $\begin{array}{c}\text { Primeiras consultas odontológicas } \\
\text { programáticas }\end{array}$ & $\begin{array}{c}\text { Cobertura de primeira consulta odontológica } \\
\text { programática (\%) }\end{array}$ \\
\hline 2006 & 10.824 .242 & 301.309 & 2,8 \\
2007 & 10.882 .121 & 254.729 & 2,3 \\
2008 & 10.940 .311 & 266.404 & 2,4 \\
2009 & 11.057 .629 & 426.232 & 3,8 \\
\hline
\end{tabular}

Fonte: Secretaria Municipal de Saúde de São Paulo.

As equipes de saúde bucal (ESB) na Estratégia de Saúde da Família do município começaram a ser cadastradas junto ao Ministério da Saúde, a partir de 2001, com 13 equipes. Este número se manteve inalterado até 2007, quando o número se ampliou para 72 ESB. Em dezembro de 2009, havia 129
ESB cadastradas no MS, sendo 71 na modalidade I (cirurgião-dentista e auxiliar em saúde bucal) e 58 na modalidade II (cirurgião-dentista, técnico em saúde bucal e auxiliar em saúde bucal). A cobertura populacional potencial é apontada na Tabela 3 (Brasil, 2010).

Tabela 3 - Número de equipes de saúde bucal na Estratégia de Saúde da Família e cobertura populacional potencial no município de São Paulo, em 2001 e 2009.

\begin{tabular}{lcc} 
Ano & Número de equipes de saúde bucal & Cobertura populacional potencial (\%) \\
2001 & 13 & 0,8 \\
2009 & 129 & 8,2 \\
\hline
\end{tabular}

Fonte: Ministério da Saúde.

Levantamento realizado em 2008 constatou que $73,8 \%$ das crianças de 18 a 36 meses nunca foram ao cirurgião-dentista, sendo este percentual reduzido para $43,1 \%$ aos 5 anos, $17,8 \%$ aos 12 anos e $9,3 \%$ entre 15 e 19 anos. Em relação ao acesso à consulta odontológica no último ano, nas idades de 18 a 36 meses, 5, 12 e 15-19 anos, os percentuais foram, respectivamente, de 10,9\%, 31,7\%, 39,9\%, e 47,3\%.

O serviço público foi o mais utilizado para as idades de 18 a 36 meses (5,5\%), 5 anos (16,9\%) e 12 anos (32,6\%). Já na faixa etária de 15 a 19 anos, os serviços privados (37,5\%) superam os serviços públi$\cos (20,7 \%)$. Em relação aos "convênios e planos de saúde”, como locais de ocorrência da última consulta odontológica, nas idades de 18 a 36 meses, 5, 12, 1519 anos, os percentuais foram de 3,3\%, 7,9\%, 10,5\% e 14,2\% (São Paulo, 2009a).

Esta redução de utilização de serviços públicos em idades maiores havia sido constatada no Inquérito de Saúde no Município de São Paulo (ISA-Capital), em 2003. Foi relatada uma baixa oferta de serviços do SUS para tratamento odontológico individual, em especial de adultos e idosos. Outro diferencial foi escolaridade. Do total de entrevistados com o a 3 anos de escolaridade, o SUS respondeu por 30,6\% das consultas odontológicas, sendo este percentual reduzido para $18,1 \%$ de 4 a 7 anos, $9,5 \%$ de 8 a 11 e de 0,9\% em habitantes com mais de 12 anos de escolaridade (São Paulo, 2005).

O número de beneficiários de planos exclusivamente odontológicos e de outros planos com cobertura odontológica está indicado na tabela 4. 
Tabela 4 - Número de beneficiários segundo o tipo de plano de saúde no município de São Paulo, em 2000 e
2009

\begin{tabular}{lcc} 
Tipo de plano & Ano & 2009 \\
Exclusivamente odontológico & 2000 & 1.719 .790 \\
Outros planos com cobertura odontológica & 475.668 & 250.499 \\
TOTAL & 185.758 & 1.970 .289 \\
\hline
\end{tabular}

Fonte: Agência Nacional de Saúde Suplementar

O total de beneficiários de planos exclusivamente odontológicos e de outros com cobertura odontológica ampliou-se na cidade, correspondendo a uma variação de cobertura populacional de 6,3\% para $17,9 \%$ da população do município, no período de março de 2000 a setembro de 2009 (São Paulo, 2010b). Segundo a forma de contratação dos planos exclusivamente odontológicos, por competência em setembro de $2009,11,2 \%$ eram individuais ou familiares, 87,8\% coletivos e 1,0\%, não informados. Quanto à época de contratação, 92,9\% eram classificados como planos novos, cujos contratos foram celebrados na vigência da Lei Federal nº 9.656/98; $6,1 \%$ planos antigos (celebrados antes da entrada em vigência da referida legislação); e 1,0\% não informados. 0 crescimento dos planos exclusivamente odontológicos no período analisado ( 9 anos e 6 meses) foi da ordem de $261,6 \%$, o que corresponde a um crescimento médio anual de $27,5 \%$.

\section{Discussão}

O acesso e a utilização de serviços de saúde dependem de um conjunto de fatores, divididos em determinantes da oferta e da demanda (Barata, 2008). Os determinantes da utilização dos serviços de saúde podem ser relacionados à necessidade de saúde (morbidade, gravidade e urgência da doença); aos usuários (características demográficas, socioeconômicas, culturais e psíquicas); aos prestadores de serviços (características demográficas, tempo de graduação, especialidade, experiência profissional, tipo de prática, forma de pagamento); à organização (recursos disponíveis, características da oferta, modo de remuneração, acesso geográfico e social); e à política (tipo de sistema de saúde, financiamento, tipo de seguro de saúde, quantidade, tipo de distribuição dos recursos, legislação e regulamentação profissional e do sistema) (Travassos e Martins, 2004).

O acesso a serviços de saúde é um conceito importante para explicar seu padrão de uso. As condições de acesso refletem as características da oferta de serviços, que podem facilitar ou dificultar a capacidade dos indivíduos utilizarem serviços de saúde de acordo com as suas necessidades (Barreto Junior e col., 20o8). Pelo lado da demanda, o estado ou necessidade de saúde é o principal determinante de uso dos serviços. A necessidade percebida, entendida como a identificação de um problema pelo usuário é o elemento mais importante da demanda, sobrepondo-se a outras considerações de ordem demográfica e social (Barata, 2008).

As maiores desigualdades no acesso e utilização de serviços odontológicos foram encontradas nos grupos de menor acesso ou utilização, sendo que a participação do Sistema Único de Saúde nos atendimentos odontológicos é muito mais baixa que na atenção médica (Barros e Bertoldi, 2002). 0 país convive com graves dificuldades no acesso à assistência odontológica, como constatam as Pesquisas Nacionais por Amostra de Domicílios (PNAD), sobre acesso e utilização de serviços de saúde, conduzidas pelo Instituto Brasileiro de Geografia e Estatística (IBGE) em 1998, 2003 e 2008.

Em 1998, 18,7\% dos entrevistados declararam nunca ter consultado um cirurgião-dentista, o que equivalia a 29,6 milhões de brasileiros. Destes, $85,6 \%$ eram crianças menores de 4 anos, 20 , $5 \%$ homens e 32,0\% população residente em áreas rurais. A porcentagem dos que declararam nunca ter consultado cirurgião-dentista era nove vezes superior 
entre as pessoas com renda de até 1 salário mínimo, quando comparadas com as que recebiam mais de 20 salários mínimos (IBGE, 200o).

Durante os anos 1990, houve expressivo declínio das ações de saúde bucal no SUS, com o Ministério da Saúde atribuindo baixíssima prioridade para a Política Nacional de Saúde Bucal, a partir do governo Itamar Franco. Essa seguiu irrelevante durante os anos 1990, atravessando o governo de Fernando Henrique Cardoso (1995-2002), sem qualquer impulso significativo ou renovação (Narvai e Frazão, 2008). É nesse contexto que se processou a promulgação da Lei Federal no 9.656/98 e a criação da Agência Nacional de Saúde Suplementar (ANS), através da Lei Federal no 9.961/oo. Trataram-se dos primeiros instrumentos para a regulação da saúde suplementar no Brasil. A partir daí, criaram-se os mecanismos para ordenar o mercado de prestação de serviços privados de saúde através de empresas denominadas operadoras de planos e seguros de saúde.

Embora antes da edição da Portaria GM n ${ }^{0}$ 1.444/200o, alguns municípios tivessem, com recursos próprios ainda nos anos 1990, incorporado a assistência odontológica nas atividades do então chamado "Programa" de Saúde da Família, a referida portaria viabilizou o aporte de recursos federais para implantar equipes de modalidade I e modalidade II. Além disso, fixou valores de um incentivo adicional em parcela única por equipe implantada, para aquisição de instrumental e equipamentos odontológicos (Brasil, 200o). Em março de 2001, foram implantadas as primeiras equipes de modalidade I e II. Entretanto, a expansão foi tímida, pois em dezembro de 2002, havia 3.819 equipes modalidade I e 442 equipes de modalidade II implantadas, com uma cobertura populacional de $15,2 \%$ da população.

Após a posse do Presidente Luís Inácio Lula da Silva, abriu-se a possibilidade de aglutinação de um projeto comum às forças democráticas que gestavam a saúde bucal como direito de cidadania (Costa e col. 2006). Em 2003, o IBGE realizou pesquisa sobre acesso e utilização de serviços de saúde. A parcela de brasileiros que nunca foi ao cirurgião-dentista era de $15,9 \%$, o que correspondia a 27,9 milhões de pessoas. A proporção dos que nunca consultaram um cirurgião-dentista era de $81,8 \%$ entre os meno- res de cinco anos, de $17,5 \%$ nos homens e $14,3 \%$ nas mulheres, de $13,6 \%$ na população urbana e $28 \%$ na rural. Enquanto 31\% da população com rendimento mensal familiar de até um salário mínimo nunca haviam feito uma consulta odontológica, a proporção diminuía para 3\% entre os que tinham rendimento superior a 20 salários mínimos (IBGE, 2005).

Em janeiro de 2004, foi lançado o documento “Diretrizes da Política Nacional de Saúde Bucal". As diretrizes constituem o eixo político básico para a reorientação das concepções e das práticas no campo da saúde bucal, sendo conhecida sob a marca "Brasil Sorridente". A reorganização da atenção em saúde bucal é proposta em todos os níveis de atenção, sendo a reorientação do modelo centrada no eixo do cuidado. Para organizar este modelo são propostas "linhas do cuidado" para a criança, adolescente, adulto e idoso. No âmbito da assistência, essas diretrizes apontam para a ampliação e qualificação da atenção básica, com acesso disponível para todas as faixas etárias e a oferta de mais serviços, de nível secundário e terciário, visando à integralidade da atenção (Brasil, 2004).

A pesquisa sobre o acesso e utilização de serviços de saúde do IBGE em 2008 constatou que a parcela de brasileiros que nunca foi ao cirurgião-dentista caiu para $11,7 \%$. A proporção dos que nunca consultaram um cirurgião-dentista era de $77,9 \%$ entre os menores de cinco anos, de 12,8\% nos homens e 10,6\% nas mulheres, de 10\% na população urbana e 20\% na rural. Enquanto 98,0\% das pessoas pertencentes à classe de rendimento domiciliar per capita de mais de 5 salários mínimos já tinham se consultado com um cirurgião-dentista, o percentual foi de $71,3 \%$ para aqueles na classe de até $25 \%$ do salário mínimo (IBGE, 2010).

O município de São Paulo ingressou na primeira década do século XXI enfrentando uma grave crise no setor saúde, após as gestões dos Prefeitos Paulo Maluf (1993-1996) e Celso Pitta (1997-200o). A partir de 1996, ocorreu a implantação do Plano de Atendimento à Saúde (PAS), com a divisão do município em módulos gerenciados por cooperativas privadas. Como consequências do PAS, destacaram-se a redução no atendimento direto por parte do município, a elevação nos gastos e a incorporação da lógica da empresa privada nas relações trabalhistas (Jun- 
queira, 2002).

Com a posse da Prefeita Marta Suplicy, em 2001, estabeleceram-se cinco frentes de atuação: a reconstrução da Secretaria Municipal de Saúde (SMS); a distritalização; a municipalização; a modernização gerencial; e a adoção do PSF como estratégia de reorganização da atenção básica (Silva, 2004). Em 2003, a gestão reconhecia o déficit nas equipes de saúde bucal, principalmente na presença de pessoal auxiliar, atribuído aos oito anos das gestões Maluf e Pitta (São Paulo, 2003).

Em 2003, inquérito de saúde conduzido na cidade (ISA-Capital) sobre morbidade referida e utilização de serviços de saúde identificou que a menor utilização de serviços do SUS ocorreu para consulta odontológica (11,9\%), sendo superada por prevenção de câncer de próstata (25,5\%), exame físico da mama (37,6\%), mamografia (37,8\%), papanicolau $(52,3 \%)$, morbidade 15 dias $(59,5 \%)$, pré-natal $(63,3 \%)$, parto $(63,4 \%)$ e puericultura $(70,7 \%)$.

Os dois principais tipos de serviços utilizados para a realização de consultas odontológicas pelos não-atendidos pelo SUS foram o serviço privado $(82,1 \%)$ e os sindicatos $(3,6 \%)$. Em relação ao procedimento "consulta odontológica", as três principais fontes de financiamento foram o gasto próprio $(57,8 \%)$, convênio da empresa $(6,9 \%)$ e plano de saúde (4,2\%) (São Paulo, 2005). Em relação ao tipo de profissional procurado no serviço de saúde, 73,7\% dos entrevistados relataram ter procurado médicos, 5,1\% farmacêuticos, e o,7\% cirurgiões-dentistas (São Paulo, 2007a).

Embora tenha havido aumento na oferta de serviços odontológicos públicos na primeira década do século XXI no município, esta foi insuficiente para ter impacto na melhoria do acesso da população. Robles (2008) destaca que as Diretrizes da Política Municipal de Saúde Bucal de São Paulo estavam alinhadas às Diretrizes da Política Nacional de Saúde Bucal, mas vinham sendo implantadas com dificuldades.

Dois fenômenos ocorridos na primeira década do século XXI ilustram as demandas da população por acesso à assistência odontológica pública na cidade. No processo de elaboração do Orçamento Participativo (OP) da Prefeitura Municipal de São Paulo para o ano de 2002, constatou-se que a saúde bucal foi a segunda especialidade mais exigida pelo público que participou das plenárias, só sendo superada pelo Programa de Saúde da Família (PSF) (São Paulo, 2001). Junqueira (2002) destaca que a Secretaria Municipal de Saúde, no início do governo Marta Suplicy em 2001, assumia o PSF como a estratégia estruturante do SUS. Mesmo nesta conjuntura (São Paulo, 2001), a demanda por saúde bucal superou todas as outras especialidades médicas, como saúde da mulher $\left(11^{\mathrm{a}}\right)$; do idoso $\left(12^{\mathrm{a}}\right)$; de álcool, drogas e dependência ( $15^{\mathrm{a}}$ ); da criança e do adolescente (19 ${ }^{\mathrm{a}}$ ); da saúde mental (20 ${ }^{a}$ ) de portadores de necessidades especiais $\left(21^{\mathrm{a}}\right)$; de assistência farmacêutica $\left(23^{\mathrm{a}}\right)$; de saúde do trabalhador $\left(41^{\mathrm{a}}\right)$; e de prevenção e combate às doenças sexualmente transmissíveis (DST)/AIDS (44 ${ }^{\mathrm{a}}$.

Ao se analisar o perfil socioeconômico dos participantes das assembleias do OP em 2002, verificou-se que a maioria era de mulheres, na faixa etária de 35 a 44 anos de idade, $2^{\circ}$ grau incompleto ou completo, renda familiar de 2 a 5 salários mínimos, moradora da periferia e pertencente a algum movimento de caráter comunitário-associativo (Sánchez, 2002).

Pesquisa realizada pela União de Movimentos Populares de Saúde (UMPS) nos meses de dezembro de 2002 e janeiro de 2003 , sobre atendimentos conseguidos e não conseguidos ( $n=1.921$ ) em 221 Unidades Básicas de Saúde (UBS) do município, constatou como principal problema para os usuários, o acesso ao "tratamento de dentes", com 50,5\% dos entrevistados relatando não ter conseguido acesso a serviços de assistência odontológica nas UBS da capital. Para se ter uma ideia da dificuldade do acesso à assistência odontológica, em relação às outras demandas, 56,5\% dos entrevistados se consultaram com psicólogos, $82 \%$ tiveram acesso a vacinas e $88 \%$ receberam controle de pressão (UMPS, 2003)..

É neste contexto que se verifica a vigorosa expansão no setor de saúde suplementar da odontologia de mercado, na cidade. Este crescimento pode ser atribuído à mudança no perfil profissional da odontologia, ao sofrível acesso da população a serviços de saúde bucal, ao baixo gasto das famílias com o desembolso direto para a assistência odontológica e o significativo peso dos gastos com planos privados de saúde (Vieira e Costa, 2008). 
A porcentagem da população beneficiária de planos exclusivamente odontológicos e de outras modalidades de planos com cobertura odontológica no município de São Paulo em setembro de 2009 $(17,9 \%)$ era superior a do Estado de São Paulo e a do Brasil. 0 número de beneficiários desses planos no Estado de São Paulo era de 5.991.298 em setembro de 2009, o que correspondia a 14,5\% da população. No Brasil, o total de beneficiários era de 15.833.499, o que equivalia a $8,3 \%$ da população. 0 espaço para crescimento dos planos odontológicos é bastante grande, caso sejam mantidas as condições estruturais que permitem prever uma grande demanda futura a ser suprida, em razão das deficiências das políticas públicas de promoção e atenção à saúde bucal (Pietrobon e col., 2008).

Manfredini (2009a) discute o potencial do mercado de odontologia suplementar na sociedade brasileira. Mesmo com o consistente crescimento verificado na primeira década do século XXI, o número de beneficiários ainda permanece distante do total de beneficiários dos planos assistenciais médicos. Há uma distância significativa entre os 12,3 milhões de usuários de planos odontológicos e os 41,9 milhões de planos de assistência médica com ou sem odontologia. Como não há identificação única, pode ocorrer de existirem beneficiários duplicados, caso possuam mais de um plano de saúde. Este mercado potencial é objeto de variadas projeções, por parte do segmento empresarial. O Sindicato Nacional das Empresas de Odontologia de Grupo (SINOG) estima que os planos odontológicos possam beneficiar mais de 40 milhões de usuários nos próximos cinco anos (Oliveira e Leite, 2009). Uma empresa de medicina de grupo projeta em quase 30 milhões o número de novos beneficiários para o setor de planos de saúde odontológicos (Spagnuolo, 2009).

Entretanto, para um país com 191 milhões de habitantes, ficam evidentes as limitações desta modalidade, típica da odontologia de mercado, para prover assistência odontológica para todos os brasileiros. Para Narvai (2002) "odontologia de mercado" corresponde a um modelo de atenção em saúde bucal que se caracteriza pela "prática centra- da na assistência odontológica ao indivíduo doente, realizada com exclusividade por um sujeito individual - o cirurgião-dentista - no restrito ambiente clínico-cirúrgico".

Assim, seja pelas limitações de cobertura próprias deste modelo no contexto brasileiro, marcado por notória iniquidade assistencial, seja pelas contradições desse modelo com os princípios de universalidade, equidade e intersetorialidade, com ênfase na promoção da saúde, ressalta-se a necessidade de dar continuidade e expandir o Brasil Sorridente, reconhecendo-o como política de Estado e não apenas de um governo e, sobretudo, buscar envolver as esferas estaduais e municipais na ampliação dos serviços públicos odontológicos.

\section{Considerações Finais}

O crescimento do número de beneficiários de planos exclusivamente odontológicos foi expressivo no município de São Paulo, na primeira década do século XXI. Este crescimento se dá no mesmo período histórico em que o Estado brasileiro adotou uma nova Política Nacional de Saúde Bucal. As diretrizes desta política foram formuladas com a intencionalidade explícita de se constituírem no eixo político básico para a reorientação das concepções e das práticas no campo da saúde bucal. No âmbito da assistência, essas diretrizes apontam para a ampliação e qualificação da atenção básica, com acesso disponível para todas as faixas etárias e a oferta de mais serviços, de nível secundário e terciário, visando à integralidade da atenção.

Constata-se que, comparativamente ao quadro geral nacional, a situação do município de São Paulo revela precariedade no acesso ao serviço odontológico público, com reduzida oferta de serviços a adultos e idosos. Indicadores de cobertura assistencial odontológica pública no município caracterizam uma situação de grande distanciamento do princípio constitucional do acesso universal aos cuidados odontológicos. Há a necessidade de enfrentamento político e organizacional desta grave questão, realinhando a cidade de São Paulo aos preceitos da atual Política Nacional de Saúde Bucal. 


\section{Referências}

BARATA, R. B. Acesso e uso de serviços de saúde: considerações sobre os resultados da PCV 2006. São Paulo em Perspectiva, São Paulo, v. 22, n. 2, p. 19-29, 2008.

BARRETO JUNIOR, I. F. B.; FERREIRA, M. P.; SILVA, Z. P. Pesquisa de condições de vida 2006: acesso aos serviços de saúde em áreas vulneráveis à pobreza. São Paulo em Perspectiva, São Paulo, v. 22, n. 2, p. 5-18, 2008.

BARROS, A. J. D.; BERTOLDI, A. D. Desigualdades na utilização e no acesso a serviços odontológicos: uma avaliação em nível nacional. Ciência \& Saúde Coletiva, Rio de Janeiro, v. 7, n. 4, p. 709-717, 2002.

BOLETIM CEINFO ISA-CAPITAL. São Paulo: Coordenação de Epidemiologia e Informação, n. 1, jul. 2005. ISA-Capital: inquérito de saúde no município de São Paulo: uso de serviços de saúde segundo motivo de procura/procedimento realizado, tipo de serviço, fonte de financiamento e escolaridade.

BOLETIM CEINFO EM DADOS. São Paulo:

Coordenação de Epidemiologia e Informação, out. 2008.

BOLETIM CEINFO EM DADOS. São Paulo:

Coordenação de Epidemiologia e Informação, out. 2009.

BOLETIM CEINFO EM DADOS. São Paulo:

Coordenação de Epidemiologia e Informação, ano IX, n. 9, 2010.

BRASIL. Lei Federal nº 9.656/98. Dispõe sobre os planos e seguros privados de assistência à saúde. Diário Oficial da União, Brasília, DF, 4 jun. 1998. Seção 1, p.1.

BRASIL. Ministério da Saúde. Gabinete do Ministro. Portaria $n^{0} 1.444$, de 28 de dezembro de 200o. Estabelece incentivo financeiro para a reorganização da atenção à saúde bucal prestada nos municípios por meio do Programa de Saúde da Família. Diário Oficial da União, Brasília, DF, 29 dez. 200o. Seção 1, p. 85.
BRASIL. Medida Provisória nº 2177-44/2001, de 24 de agosto de 2001. Altera a Lei n ${ }^{0}$ 9.656, de 3 de junho de 1998, que dispõe sobre os planos privados de assistência à saúde e dá outras providências. Diário Oficial da União, Brasília, DF, 27 ago. 2001. Seção 1, p. 38.

BRASIL. Ministério da Saúde. Secretaria de Atenção à Saúde. Departamento de Atenção Básica. Coordenação Nacional de Saúde Bucal. Diretrizes da política nacional de saúde bucal. Brasília, DF, 2004.

BRASIL. Ministério da Saúde. Agência Nacional de Saúde Suplementar. Caderno de informação da Saúde Suplementar: beneficiários, operadoras e planos. Brasília, DF, 2009. Disponível em: <http:// www.ans.gov.br/portal/site/informacoesss/ informacoesss.asp >. Acesso em: 13 jan. 2010.

BRASIL. Ministério da Saúde. Departamento de Atenção Básica. Coordenação Nacional de Saúde Bucal. Total de equipes de saúde da família: saúde bucal no município de São Paulo. Brasília, DF, 2010.

CHAVES, S. C. L.; VIEIRA-DA-SILVA, L. M. Atenção à saúde bucal e a descentralização da saúde no Brasil: estudo de dois casos exemplares no Estado da Bahia. Cadernos de Saúde Pública, Rio de Janeiro, v. 23, n. 5, p. 1119-1131, 2007.

CONSELHO FEDERAL DE ODONTOLOGIA. Dados estatísticos: cirurgiões-dentistas. Rio de Janeiro, 2010. Disponível em: <http://cfo.org.br/servicose-consultas/Dados-estatisticos/?elemento=profis sionais\&categoria $=\mathrm{CD} \& \mathrm{cro}=$ Todos\&municipio $=>$. Acesso em: 14 maio 2010.

COSTA, J. F. R.; CHAGAS, L. D.; SILVESTRE, R. M. A política nacional de saúde bucal do Brasil: registro de uma conquista histórica. Brasília, DF: Organização Pan-Americana da Saúde, 2006. (Série técnica desenvolvimento de sistemas e serviços de saúde, 11).

EMPLASA - EMPRESA PAULISTA DE PLANEJAMENTO METROPOLITANO. Indicadores metropolitanos: Região Metropolitana de São Paulo. São Paulo, 2007. 
FUNDAÇÃO SEADE - FUNDAÇÃO SISTEMA

ESTADUAL DE ANÁLISE DE DADOS. A cidade

comemora 456 anos com mudanças no perfil do

paulistano. SP Demográfico, São Paulo, v. 10, n. 1, p. 1-4, 2010.

IBGE - INSTITUTO BRASILEIRO DE GEOGRAFIA E ESTATÍSTICA. Acesso e utilização de serviços de saúde: Pesquisa Nacional por Amostra de Domicílios, 1998. Rio de Janeiro, 2000.

IBGE - INSTITUTO BRASILEIRO DE GEOGRAFIA E ESTATÍSTICA. Pesquisa Nacional por Amostra de Domicílios 2003: corpo básico e suplemento acesso e utilização de serviços de saúde. Rio de Janeiro, 2005.

IBGE - INSTITUTO BRASILEIRO DE GEOGRAFIA E ESTATÍSTICA. Pesquisa Nacional por Amostra de Domicílios 2008: um panorama da saúde no Brasil: acesso e utilização dos serviços, condições de saúde e fatores de risco e proteção à saúde. Rio de Janeiro, 2010.

JUNQUEIRA, V. Saúde na cidade de São Paulo (1989 a 200o). São Paulo: Polis: PUC-SP, 2002.

LOUVISON, M. C. P. et al. Desigualdades no uso e acesso aos serviços de saúde entre idosos do município de São Paulo. Revista de Saúde Pública, v. 42, n. 4, p. 733-740, 2008.

MANFREDINI, M. A. Características da indústria de equipamentos odontológicos e de produtos para higiene bucal no Brasil entre 1990 e 2002. 2006. Dissertação (Mestrado em Ciências) - Coordenadoria de Controle de Doenças da Secretaria de Estado da Saúde de São Paulo, São Paulo, 2006.

MANFREDINI, M. A. Atenção à saúde bucal no Brasil e perspectivas para o setor suplementar de saúde. In: AGÊNCIA NACIONAL DE SAÚDE SUPLEMENTAR. Planos odontológicos: evolução, desafios e perspectivas para a regulação da saúde suplementar. Rio de Janeiro, 2009a. p. 17-29.

MANFREDINI, M. A. Mercado de produtos de higiene bucal no Brasil: uma análise crítica. In: MOYSÉS, S. J.; GROISMAN, S. Programa de Atualização em Odontologia Preventiva e Saúde Coletiva. Porto Alegre: Artmed: Panamericana, 2009b. p. 61-87.
NARVAI, P. C. Odontologia e saúde bucal coletiva. São Paulo: Ed. Santos, 2002.

NARVAI, P. C., FRAZÃO, P. Políticas de saúde bucal no Brasil. In: MOYSÉS, S. T.; KRIGER, L.; MOYSÉS, S. J. (Coord.). Saúde bucal das famílias: trabalhando com evidências. São Paulo: Artes Médicas, 2008. p. 1-20.

OLIVEIRA, R.; LEITE, V. S. Planos odontológicos desaceleram expansão. Gazeta Mercantil, São Paulo, 14 abr. 2009. p. C6.

PESSOTO, U. C. et al. Desigualdades no acesso e utilização dos serviços de saúde na Região Metropolitana de São Paulo. Ciência \& Saúde Coletiva, Rio de Janeiro, v. 12, n. 2, p. 351-362, 2007.

PIETROBON, L. et al. Planos de assistência à saúde: interfaces entre o público e o privado no setor odontológico. Ciência \& Saúde Coletiva, Rio de Janeiro, v. 13, n. 5, p. 1589-1599, 2009.

RIBEIRO-SOBRINHO, C.; SOUZA, L. E. P. F.; CHAVES, S. C. L. Avaliação da cobertura do Serviço Odontológico da Polícia Militar da Bahia em Salvador, Bahia, Brasil. Cadernos de Saúde Pública, Rio de Janeiro, v. 24, n. 2, p. 295-302, 2008. ROBLES, L. P. Atenção à saúde bucal no município de São Paulo de 2005 a 2007. 2008. Tese (Doutorado em Saúde Pública) - Faculdade de Saúde Pública da Universidade de São Paulo, São Paulo, 2008.

ROLNIK, R. São Paulo, novo século: uma nova geografia? In: GARIBE, R.; CAPUCCI, P. Gestão local nos territórios da cidade: ciclo de atividades com as subprefeituras. São Paulo: Mídia Alternativa: Secretaria Municipal das Subprefeituras, 2004. p. 25-31.

SÁNCHEZ, F. R. Orçamento participativo: teoria e prática. São Paulo: Cortez, 2002.

SÃO PAULO. Secretaria de Governo Municipal. Coordenação do Orçamento Participativo. Propostas da área de saúde para o OP-2002. São Paulo, 2001.

SÃO PAULO. Secretaria Municipal de Saúde. Revista Saúde, São Paulo, v. 1, abr, 2003. 
SÃO PAULO. Secretaria Municipal da Saúde. Coordenação de Epidemiologia e Informação. Inquérito de Saúde no Município de São Paulo ISACapital 2003: principais resultados - estilo de vida, morbidade referida, uso de serviços, autoavaliação de Saúde e temas especiais. São Paulo: 2007a.

SÃO PAULO. Secretaria Municipal do Trabalho. Atlas do trabalho e desenvolvimento da cidade de São Paulo. São Paulo, 2007b.

SÃO PAULO. Secretaria Municipal de Saúde. Coordenação da Atenção Básica. Área Técnica de Saúde Bucal. Levantamento epidemiológico em saúde bucal: cidade de São Paulo, 2008-2009: resumo da primeira fase: crianças e adolescentes. São Paulo, 2009.

SÃO PAULO. Secretaria Municipal de Saúde. Saúde antecipa cumprimento de metas da Agenda 2012. São Paulo, 2010a. Disponível em: <http://www. prefeitura.sp.gov.br/cidade/secretarias/saude/ noticias/?p=13863>. Acesso em: 10 jan. 2010.

SÃO PAULO. Secretaria Municipal de Saúde. População do município de São Paulo. São Paulo: 201ob. Disponível em: <http://ww2.prefeitura. sp.gov.br/cgi/deftohtm.exe?secretarias/saude/ TABNET/POP/pop.def>. Acesso em: 13 jan. 2010b.
SÃO PAULO. Secretaria Municipal de Saúde. Saúde bucal direito de cidadania! São Paulo, 2010c.

SILVA, Z. P. A política municipal de saúde (20012003). São Paulo: Instituto Pólis: PUC-SP, 2004.

SPAGNUOLO, S. Setor odontológico vislumbra mercado da AL. América Economia, São Paulo. Disponível em: <http://www.americaeconomia. com.br>. Acesso em: 9 jun. 2009.

TRAVASSOS, C.; MARTINS, M. Uma revisão sobre os conceitos de acesso e utilização de serviços de saúde. Cadernos de Saúde Pública, Rio de Janeiro, v. 20, p. S19o-S198, 2004. Suplemento 2.

USUÁRIOS estão insatisfeitos com a demora, falta de profissionais e medicamentos. Jornal da UMPS, São Paulo, ago. 2003. p. 2-3.

VIEIRA, C., COSTA, N. R. Estratégia populacional e mimetismo empresarial: os planos de saúde odontológicos no Brasil. Ciência \& Saúde Coletiva, Rio de Janeiro, v. 13, n. 5, p 1579-1588, 2008.

Recebido em: $11 / 11 / 2010$

Reapresentado em: 03/11/2011

Aprovado em: 08/12/2011 